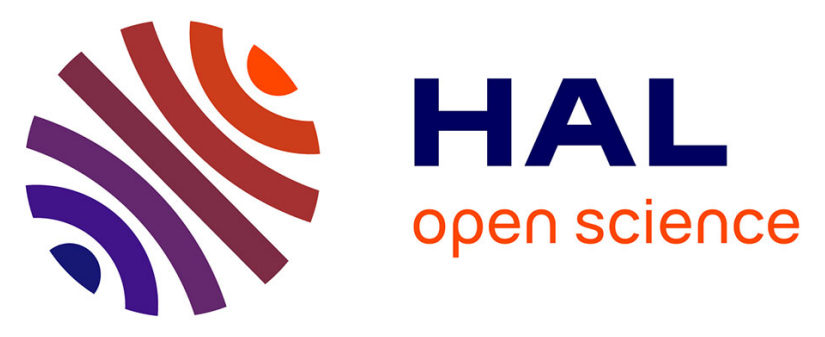

\title{
Mobility Prediction in Vehicular Networks: An Approach through Hybrid Neural Network under Uncertainty
}

\author{
Soumya Banerjee, Samia Bouzefrane, Paul Mühlethaler
}

\section{To cite this version:}

Soumya Banerjee, Samia Bouzefrane, Paul Mühlethaler. Mobility Prediction in Vehicular Networks: An Approach through Hybrid Neural Network under Uncertainty. International Conference on Mobile Secure and Programmable Networking (MSPN 2017), pp.195-217, Series Springer LNCS 10566, Jun 2017, Paris, France. pp.178-194, 10.1007/978-3-319-67807-8_14 . hal-02425156

\section{HAL Id: hal-02425156 https://hal.science/hal-02425156}

Submitted on 29 Dec 2019

HAL is a multi-disciplinary open access archive for the deposit and dissemination of scientific research documents, whether they are published or not. The documents may come from teaching and research institutions in France or abroad, or from public or private research centers.
L'archive ouverte pluridisciplinaire HAL, est destinée au dépôt et à la diffusion de documents scientifiques de niveau recherche, publiés ou non, émanant des établissements d'enseignement et de recherche français ou étrangers, des laboratoires publics ou privés. 


\title{
Mobility Prediction in Vehicular Networks : An Approach through Hybrid Neural Networks under Uncertainty
}

\author{
*Soumya Banerjee ${ }^{\dagger}$ Samia Bouzefrane ${ }^{\ddagger}$ Paul Muhethaler
}

\begin{abstract}
Conventionally, the exposure regarding knowledge of the inter vehicle link duration is a significant parameter in Vehicular Networks to estimate the delay during the failure of a specific link during the transmission. However, the mobility and dynamics of the nodes is considerably higher in a smart city than on highways and thus could emerge a complex random pattern for the investigation of the link duration, referring all sorts of uncertain conditions. There are existing link duration estimation models, which perform linear operations under linear relationships without imprecise conditions. Anticipating, the requirement to tackle the uncertain conditions in Vehicular Networks, this paper presents a hybrid neural network-driven mobility prediction model. The proposed hybrid neural network comprises a Fuzzy Constrained Boltzmann machine $(F C B M)$, which allows the random patterns of several vehicles in a single time stamp to be learned. The several dynamic parameters, which may make the contexts of Vehicular Networks uncertain, could be vehicle speed at the moment of prediction, the number of leading vehicles, the average speed of the leading vehicle, the distance to the subsequent intersection of traffic roadways and the number of lanes in a road segment. In this paper, a novel method of hybrid intelligence is initiated to tackle such uncertainty. Here, the Fuzzy Constrained Boltzmann Machine (FCBM) is a stochastic graph model that can learn joint probability distribution over its visible units (say $\mathrm{n}$ ) and hidden feature units (say $\mathrm{m}$ ). It is evident that there must be a prime driving parameter of the holistic network, which will monitor the interconnection of weights and biases of the Vehicular Network for all these features. The highlight of this paper is that the prime driving parameter to control the learning process should be a fuzzy number, as fuzzy logic is used to represent the vague and and uncertain parameters. Therefore, if uncertainty exists due to the random patterns
\end{abstract}

* Soumya Banerjee,Visiting Prof. Conservatoire National des Arts et Metiers, Paris Cedex 03, France, Birla Institute of Technology, Mesra, India, e-mail:soumyabanerjee@bitmesra.ac.in ${ }^{\dagger}$ Samia Bouzefrane, Conservatoire National des Arts et Metiers, Paris Cedex 03, France,

${ }^{\ddagger}$ Paul Muhlethaler, Eva team, INRIA 2 Rue Simone IFF, 75012 Paris, France, 
caused by vehicle mobility, the proposed Fuzzy Constrained Boltzmann Machine could remove the noise from the data representation. Thus, the proposed model will be able to predict robustly the mobility in VANET, referring any instance of link failure under Vehicular Network paradigm.

Keywords: Vehicular Network, Mobility Prediction, Link Failure, Fuzzy Constrained Boltzmann Machine, VANET, Uncertainty.

\section{Introduction}

With the increase of wireless networks and the growing trends towards the Internet of Things (IoT), vehicular communication is being viewed from different perspectives. These include the road safety and traffic management [1]. However, scenarios of vehicular networks are becoming more complex as several dynamic parameters of vehicles are being introduced : vehicle speed at the moment of prediction, number of leading vehicles, average speed of the leading vehicles, the distance to the subsequent intersection and the numbers of lanes in a road segment. The problem is thus more realistic and several research initiatives are already being accomplished, by considering the data obtained relating short-term vehicle movement [2][3]. The reliability of contexts, variables in different road intersections, different traffic scenarios and inter-vehicle link duration offer challenges to formulate the prediction model [4]. In addition to, a substantial number of research initiatives concern probabilistic modeling of vehicles which infer immediate future locations. Even so, it has been observed that to configure a robust and intelligent vehicular networks [5], each tiny parameter such as road intersection problem parameters can be handled with an effective group scheduling of vehicles. Thus those intelligent neuro-fuzzy (neural-network and fuzzy logic driven) models becoming more adaptive to suit different traffic conditions [6]. Inspired by such models [7] [8], this paper proposes a Fuzzy Constrained Boltzmann Machine (FCBM). This is a stochastic graph model and can learn joint probability distributions over certain time units with many existing as well as hidden features of different vehicular network environments. The relevance of proposed approach is two fold: firstly, the class of Boltzmann machine is a specialized class of deep learning algorithm and no such model currently exists. Moreover, conventional deep learning models are being controlled with visible and hidden features of problem domain. In this case, an uncertain relationship is represented with these inherent uncertainty as a fuzzy number. Thus, the constraints of relationships between the features should be driven by fuzzy logic and this could serve to train the Boltzmann machine to infer smarter decision about mobility predictions in vehicular networks. We develop the simulation and experimental model and test it with the corresponding data set. Several interesting observations have been obtained. The analysis shows that a hybrid intelligent model is required, where uncertainty and non-linear optimal conditions persist. The remaining part of the paper has been organized as follows: Section 2 briefly mentions the most relevant intelligent models deployed for vehicular networks under different conditions. Section 3 develops a mathe- 
matical formulation of the proposed approach, and outlines the role of auxiliary functions for modeling fuzzy logic in Boltzmann scheme in Section 3.1. Section 4 provides a short introduction to the highlights conventional Boltzmann machine and its relevance to hybrid neural networks. Section 4.1 gives details of simulation and the corresponding results comparing them to the available data set. Finally, Section 5 highlights about the contributions and mentions future research directions in the field.

\section{Related Work}

Very few core research implementations are available using different computational intelligence schemes in vehicular networks for mobility prediction. Most approaches (e.g. fuzzy logic and rough set) use clustering or classifications of vehicles according to their location even in boundary regions [12] [13]. However specific intersection control problems in smart cities are being treated with neuro-fuzzy learning from real traffic conditions [14]. Traffic and vehicle speed prediction have also been developed using neural networks and hidden Markov models [6]. Inspite of all the existing models, sensing techniques and prediction of mobility in vehicular networks have raised substantial research challenges. This is primarily because, none of the intelligent models could encompass diversified uncertain parameters of vehicular networks and making the predictions unrealistic. Inspired by recent studies of deep learning and machine learning approaches [15], this paper adopts a basic Boltzmann machine approach. The model is trained through fuzzy numbers, which represents different non-linear features of vehicular network as well as network connectivity overheads. The proposed model is termed as hybrid neural network.

\section{Mathematical Formulation of Proposed Model}

The following parameters are being considered, while formulating the proposed model :

- Vehicle speed $\mathrm{S}_{m}$ at the moment of prediction

- Number of leading vehicles

- Average speed of leading vehicles

- Distance (optional) to the next intersection

- Number of lanes in the road segment $\left(\mathrm{R}_{s}\right)$

These parameters are non-deterministic and lead to major concerns of uncertainty in vehicular networks. In addition, these parameters and their associated contexts can contain uncertainties. They are listed as :

- Change of vehicle Speed 
Table 1: List of Prime Variables

\begin{tabular}{cc}
\hline Parameters / Variables & Semantics \\
\hline \hline$i \in I$ & $\begin{array}{c}\text { Time interval between } \\
\text { vehicles }->\mathrm{I}=\{1,2, \ldots \ldots \mathrm{m}\}\end{array}$ \\
\hline \hline$j \in J$ & $\begin{array}{c}\text { Index of different access } \\
\text { points }(\mathrm{AP}), \text { where } \\
\mathrm{J}=\{1,2, \ldots . \mathrm{n}\}\end{array}$ \\
\hline \hline$\gamma^{1}$ & $\begin{array}{c}\text { Vehicle departure ratio } \\
\text { from source }\end{array}$ \\
\hline \hline $\mathrm{M}_{i j}$ & $\begin{array}{c}\text { Rate of mobility from } \mathrm{i} \text { to } \\
\mathrm{j}\end{array}$ \\
\hline \hline $\mathrm{L}_{i}$ & Length of time interval \\
\hline $\mathrm{x}_{i j}$ & $\begin{array}{c}\text { Mobility prediction } \\
\text { decision variable for the } \\
\end{array}$ \\
\hline \hline $\mathrm{P}_{s}$ & points i to $\mathrm{j}$ \\
\hline \hline$\alpha$ & $\begin{array}{c}\text { Prediction Scenario with } \\
\text { respect to the parameters } \\
\text { mentioned for Vehicular } \\
\text { Network }\end{array}$ \\
\hline \hline
\end{tabular}

- Different driving habits and road conditions

- Density of traffic

- Position of traffic lights

Therefore a specific objective function can be formulated.

The objective function is described, the objective function can train the proposed Boltzmann Machine through symmetric triangular Fuzzy Numbers. The inclusion of fuzzy factor sis to tackle uncertain parameters and their contexts mentioned in the previous description. We divide the approach into two major parts:

a. Initially, optimal control of the delay for vehicle:

The total vehicle delay time for whole network is:

$$
\left.\operatorname{Min} V_{D}=\left[\min \sum_{j \in J} \sum_{k=1}^{P_{S}} \sum_{i \in R_{s}} V_{i}^{j}(k)-M_{i j}(k)\right] \alpha\right]
$$

where $V_{i}^{j}(k)$ is the number of vehicles for point $\mathrm{i}$ for road section $\mathrm{R}_{s}$ at the time instance $\mathrm{k}$ and $\alpha$ is the sampling interval period for complete network coverage.

b. For this part, we assume that there must exist a non-linear optimal control of mobility, where, for training with the uncertain parameters of the vehicular network, a fuzzy number is introduced in triangular form (it signifies that the 
core function can represent at least three values of membership or certainty factor: for example: road traffic could be moderately normal, medium, strongly adequate etc.). We also observe that there could be different trends of mobility for two communicating vehicles before the communication may fail due to the predicted enhancement in the intermediate distance. Therefore, this non-linear factor can be represented with another form of exponentiation function. Thus, if the minimum value of vehicle delay under non-linear/uncertain factors is being considered, then

$$
\operatorname{Min} V_{D}=\left[\operatorname { m i n } \sum _ { j \in J } \sum _ { k = 1 } ^ { P _ { S } } \sum _ { i \in R _ { s } } \left[V_{i}^{j}(k)-\exp \left(\beta_{0}^{j}(k)\right] \alpha\right.\right.
$$

Here, $\mathrm{P}_{s}$ the prediction scenario, parameter $\beta_{0}^{j}(k)$ and $V_{i}^{j}(k)$ is the result of an auxiliary function, this will be essentially to formulate a final value of the training function for the Boltzmann Machine. In practice, Boltzmann machines are comprised of visible units, (say V), and hidden units. The visible units are those which receive information from the 'environment' (in this case it could be the traffic conditions from the road and other features derived from the traffic contexts), i.e. the training set is a set of binary vectors over the set V. The distribution over the training set is denoted as a continuous function $\mathrm{P}^{+}(V)$. Moreover the machine has an energy reference, which is modified through the positioning of interconnected weights of features during the operation. The machine may stop learning correctly, when it is scaled up to anything larger than a trivial value. There are two phases involved in Boltzmann machine training, and we switch iteratively between both of them. One is the positive phase, where the visible units' states are clamped to a particular binary state vector sampled from the training set (according to $\mathrm{P}^{+}$). The other is the negative phase, where the network is allowed to run freely. Therefore, the reference energy level of the machine should be discrete out of uncertainty factors and this switching effect from positive to negative must encompass all the membership values of uncertainty [9][10]. As in this case, the hidden features in random or urban traffic conditions are free from external interference, and their values are unknown to us. Hence, we cannot update their weights. Thus, the more membership or certainty values of hidden feature vectors are introduced, the more precise prediction can be.

In the next subsection, the structure of the auxiliary function is derived.

\subsection{Structure of the Auxiliary Function in a Vehicular Network}

Here, we refer to the structure of $\beta_{0}^{j}(k)$ :

$$
\delta_{0}^{J}(k)=\left\{k\left[\gamma_{0}^{j}(k) \oplus \gamma_{0}^{j}(k+1)\right]\right.
$$

if $\left(1 \leqslant k \leqslant P_{s}-1\right)$ and subsequently as the value of $\delta_{0}^{J}(k)$ could be treated as a fuzzy number trivially with 3 values ; if $\mathrm{k}=0$, then $\delta_{0}^{J}(k)=0$, if $\mathrm{k}=\mathrm{P}_{s}$ 
then $\delta_{0}^{J}(k)=\mathrm{P}_{s}$. The construction of Right hand side of the expression with $\gamma_{0}^{j}$ indicates the weight bias of fuzzy nmber which is additive with the instances of values $\mathrm{k}$. That means, $\mathrm{k}$ and $(\mathrm{k}+1)$ instances are considered here for formulating auxillary function and thus $\beta_{0}^{j}$.

For implementation, we also investigate the learning features of vehicular networks and it could be either simple sample function or a multiple sample function for error estimation in the final value of the training function in the Boltzmann Machine. It is known that better scaling and an error free representation will make the network learn better for the prediction of the vehicles movement. Considering all the listed parameters, multiple sample features will be suitable to make the training of the network more error free. Assuming, the multiple sample features, the final training function, say $\mathrm{X}(\mathrm{w})$, where $\mathrm{w}$ is the edge weight of the features connected in the Boltzmann Machine, can be expressed as [10] :

$$
T(w)=\frac{1}{2} \sum_{i=1}^{n} \sum_{i=1}^{m}\left(f_{c}\left(x^{i}\right)-y_{c}^{i}\right)^{2}
$$

It is clear that two terms i.e. $f_{c}\left(x^{i}\right)$ and $y_{c}^{i}$ in the expressions related to $\mathrm{T}(\mathrm{w})$ are the coefficients of the training function to be operated on feature vectors taken from vehicular network paradigm. The first one depends on the network edge weight $\mathrm{w}$ where the second one is independent of $\mathrm{w}$. Therefore, a partial derivative is derived for the final training weight $\mathrm{w}_{P s_{j}}^{k}$ for all $\mathrm{k}$ and $\mathrm{j}$.

\section{Proposed High Level Description of the Hybrid Neural Network}

The term hybrid neural network was coined from the concept that a neural network in its core form can be modified with the supporting mode of computational intelligence like fuzzy logic, specially to for the training purposes. Prior to describing the proposed model, a brief background is presented on conventional Boltzmann Machines (Fig. 1). They were one of the first examples of a neural network capable of learning internal representations, and are able to represent and (given sufficient time) solve difficult combinatoric problems [9]. The structure comprises of some visible and some hidden units. A graphical representation of an example of a Boltzmann machine is shown in fig. 1. Each undirected edge represents dependency. In this example there are 3 hidden units and 4 visible units. 
Figure 1: Basic Structure of Boltzmann Machine Network

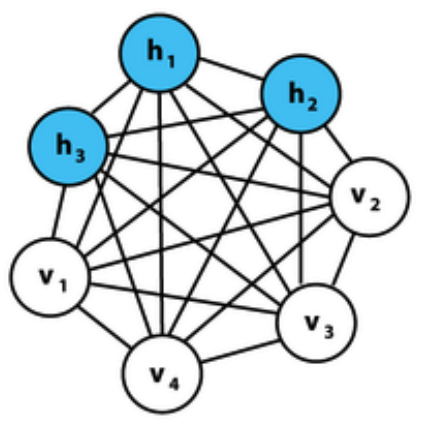

Structurally, the network can learn by the adjustment of weights and hence it finally culminates with an energy function E [9] [10]:

$$
E=-\left(\sum_{i<j} w_{i j} s_{i} s_{j}+\sum_{i} \theta_{i} s_{i}\right)
$$

where, $\mathrm{w}_{i j}$ is the connection strength between unit $\mathrm{j}$ and unit $\mathrm{i} . \mathrm{s}_{i}$ is the state, $\mathrm{s}_{i} \in\{0,1\}$ of unit $\mathrm{i}$,

$\theta_{i}$ is the bias of unit $\mathrm{i}$ in the global energy function. $\left(-\theta_{i}\right.$ is the activation threshold for the unit).

Normally, Boltzmann machines are made of two layers of neurons, with connections only between different layers and no connections within the same layer. The bottom layer (i.e. the visible one) is denoted further by a binary vector $\mathrm{v}=\left[\mathrm{v}_{1}, \mathrm{v}_{2}, . ., \mathrm{v}_{n_{v}}\right]$, in which each unit $\mathrm{v}_{i}$ is a binary unit, and where $\mathrm{n}_{v}$ is the size of $\mathrm{v}$ (i.e. the number of neurons of the visible layer). The top layer 


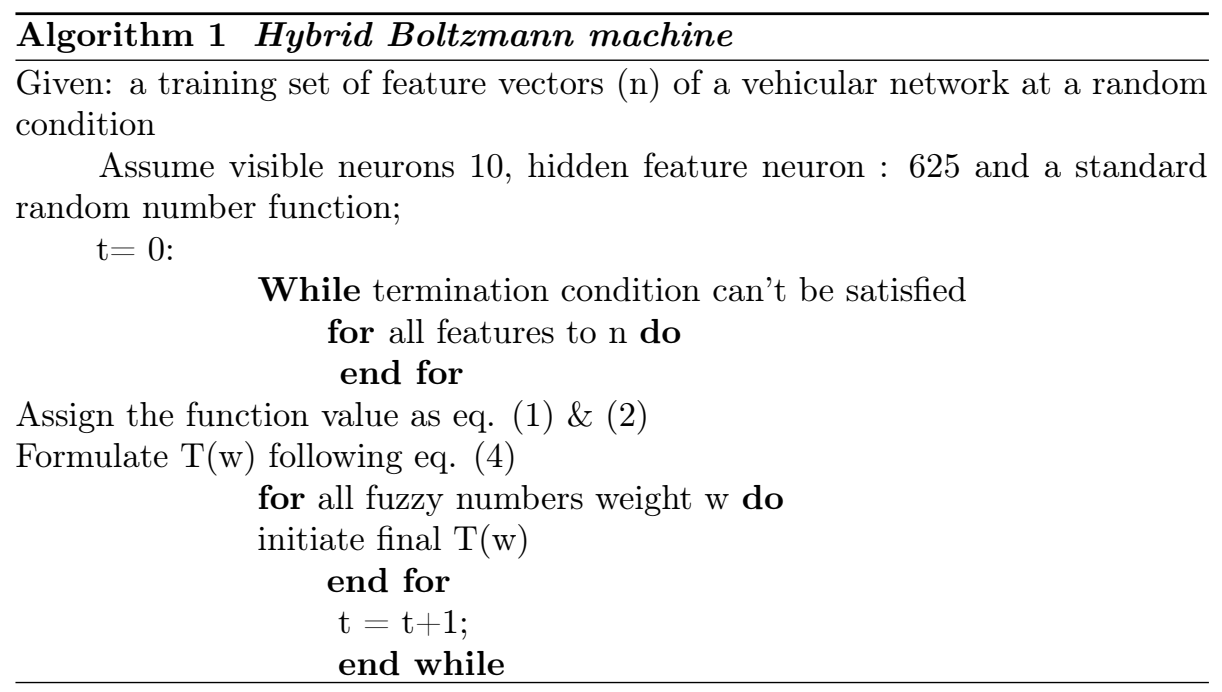

(i.e. the hidden one) is represented by the binary vector $\mathrm{h}=\left[\mathrm{h}_{1}, \mathrm{~h}_{2}, \ldots, \mathrm{h}_{n_{h}}\right]$, in which each element $h_{j}$ is binary, and where $n h$ is the size of $h$. Furthermore, each neuron from the visible layer has associated bias. The biases of the visible neurons are grouped in a vector $\mathrm{a}=\left[\mathrm{a} 1, \mathrm{a} 2, . ., \mathrm{an}_{v}\right]$. Similarly, hidden layer neurons have biases, collected in vector $\mathrm{b}=\left[\mathrm{b} 1, \mathrm{~b} 2, . ., \mathrm{bn}_{h}\right]$. The broad high level description is presented in :

In this paper, the implementations of the algorithm is done in Visual $\mathrm{C}++$. In all the settings, the momentum was set to 0.5 , the learning rate to 0.05 . We assume 10 visible and around 625 hidden (under uncertain conditions) neurons, to be trained with a fuzzy triangular function.

\subsection{Results and Analysis}

The above Fuzzy Constrained Boltzmann Machine (FCBM) algorithm is simulated in $\mathrm{VC}++5.0$ with MFC (Microsoft Foundation class) support and the algorithm is tested an available data set [11]. The $\mathrm{VC}++$ code uses two threads that read and write from the synthesized vehicular network with the function getVecMessage ( ) and sendVecMessage( ) from the library predefined as $<$ msn.h $>$. Prior to developing the desired simulation, the following propositions are made to support the simulation across the interconnected device network

- Nodes: A node is an instance of an executable and can be a sensor, actuator, processing or monitoring algorithm.

- Messages: A message is a typed data structure made up of primitive types like integer, floating point, boolean, etc., arrays of primitives and constants. Nodes communicate with each other by passing messages.

- Context of Topic: A Context of Topic is an asynchronous data transport system based on a subscribe/publish system and is identified by a name. One or more nodes are able to publish data (messages) to a topic and one 
Table 2: Analysis of Prediction Scenario with different time stamps and feature weights

\begin{tabular}{|c|c|c|c|c|c|c|c|}
\hline \multirow{4}{*}{$\begin{array}{c}\text { Wt. W } \\
\mathbf{P}_{s}=\mathbf{2 0}\end{array}$} & 0 & 2 & 3 & 4 & 5 & 6 & 7 \\
\hline & .51 .86 & .401 & .3 .61 & 001 & 001 & $\begin{array}{lll}0 & 0 & 1\end{array}$ & 001 \\
\hline & $\begin{array}{lll}1.61 \\
\end{array}$ & $\begin{array}{lll}110.8 \\
\end{array}$ & $\begin{array}{lll}1 & 1 & 1\end{array}$ & 11.5 & 11.5 & 01.7 & $\begin{array}{lllll}0 & 1 & 1\end{array}$ \\
\hline & $\begin{array}{lll}0 & 1.7\end{array}$ & 101 & 1.81 & .801 & 101 & $0 \begin{array}{lll}0 & 0 & 1\end{array}$ & 2101 \\
\hline \multirow[t]{3}{*}{$\mathbf{P}_{s}=130$} & .501 & .600 & $\begin{array}{llll}0 & 0 & 0\end{array}$ & $\begin{array}{llll}0 & 0 & 1 \\
\end{array}$ & $\begin{array}{llll}0 & 0 & 1 \\
\end{array}$ & $\begin{array}{llll}0 & 01\end{array}$ & 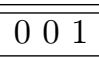 \\
\hline & $\begin{array}{llllll}0 & 1 & 0\end{array}$ & $\begin{array}{lllll}0 & 1 & 1\end{array}$ & $\begin{array}{lllll}0 & 1 & 1\end{array}$ & $\bar{~} 1.4 .6$ & $\overline{1.6 .4}$ & 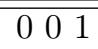 & $\begin{array}{lll}001 \\
\end{array}$ \\
\hline & $\begin{array}{lll}1.91 \\
\end{array}$ & 1.91 & 1111 & .901 & 101 & $\begin{array}{lll}1 & 0 & 1\end{array}$ & $\begin{array}{lll}11 & 1\end{array}$ \\
\hline
\end{tabular}

or more nodes can read data on that context of topic. Data is exchanged asynchronously by means of a topic and via a service. This process will help to identify more vehicular network features. Finally, this will produce a training set of vectors

- Services: A services allows nodes to communicate with each other through a synchronously communication. The service nodes are able to send a request and receive a response.

The following observation in values of weights (Fuzzy numbers) are done following the prediction scenario $\mathrm{P}_{s}$ :

The different intermediate states of the vehicular network have been demonstrated with the different weight values and there are substantial changes from weight mark iterations from 0-7. The prediction scenario $\mathrm{P}_{s}$ is also different with time stamps from 20-130 ms as shown in Fig 2. It is shown that a Fuzzy driven Boltzmann machine with different vehicle tracks and having same network overheads can predict the movement of vehicles.

The network processing overhead increases proportionally with an increase in vehicle density for all types of highway road/tracks as shown in Fig. 2. The network processing overhead in the scheme is higher because of the additional features incurred for the tasks such as accident zone identification, travel direction identification, risk factor assignment and prioritization of emergency vehicles like fire services or ambulances.

Prediction is more accurate after the first two tracks (red and blue) are being for training following the weight values shown in Table 2. The green and blue curve of the track shows certain steady value with all the features, however for prediction scenario $\mathrm{P}_{s}$ the red track diminished after certain iterations. 
Figure 2: Movement Track

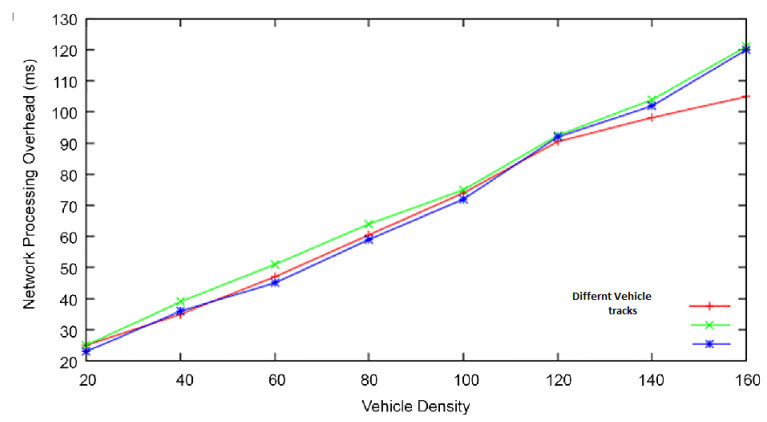

Following the data set in [11], the second part of the simulation is shown. In this case, to identify the different types of vehicles, the simulation time differs depending on the weighted feature w. However, the average vehicle density alters considerably, with respect to average connectivity distance. The other part of simulation is also done with the number and types of vehicles. The impact is one of the important parameter to understand the prediction error analysis. 
Figure 3: Impact of Vehicle acceleration and simulation time for prediction

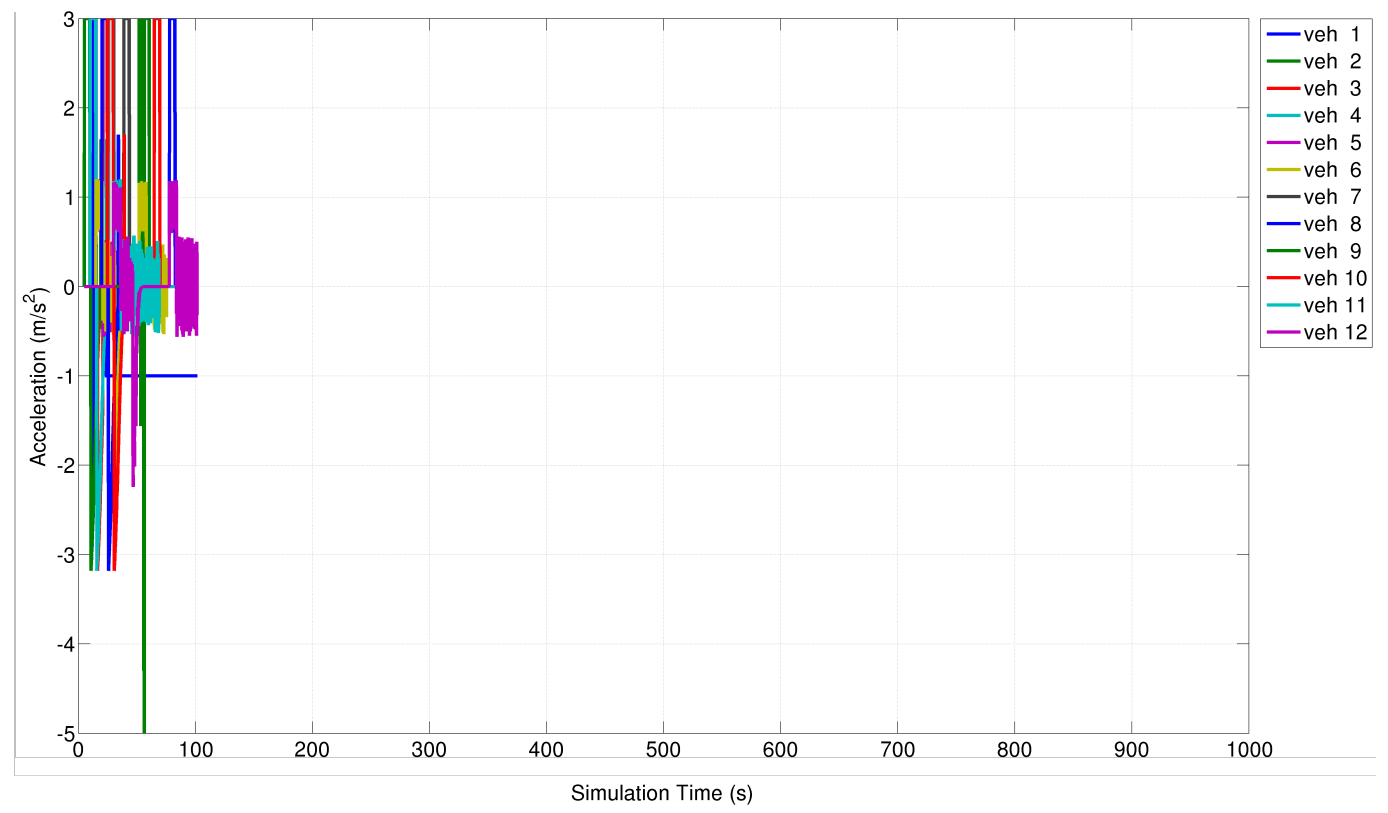

The plots shown in fig. 3 are closely analogous to the parameters studied: acceleration of 12 similar vehicles are being considered and the simulation time is calculated. The simulation code developed for the Boltzmann machine with fuzzy numbers as constraints, is shown in appendix, and following the code, the RMS value of the simulation performed on the data set [11] is given in terms of vehicle mobility predictions. The values collected for the phases of delivery and acknowledgement and the total time of the iteration have also been presented. For the acknowledgment phase, there are the minimum and maximum time used for the message and the number of conditional variables of the messages for vehicles through FCBM are also given. We also observe that the phases of configuring the Boltzmann machine with 10 visible and 625 hidden units can be kept as maximum to train the network. When more than 625 uncertain features from traffic conditions exist, the prediction time differs randomly, even after successful training with fuzzy triangular values. 
Figure 4: Restricted Vehicles with uncertain Gap

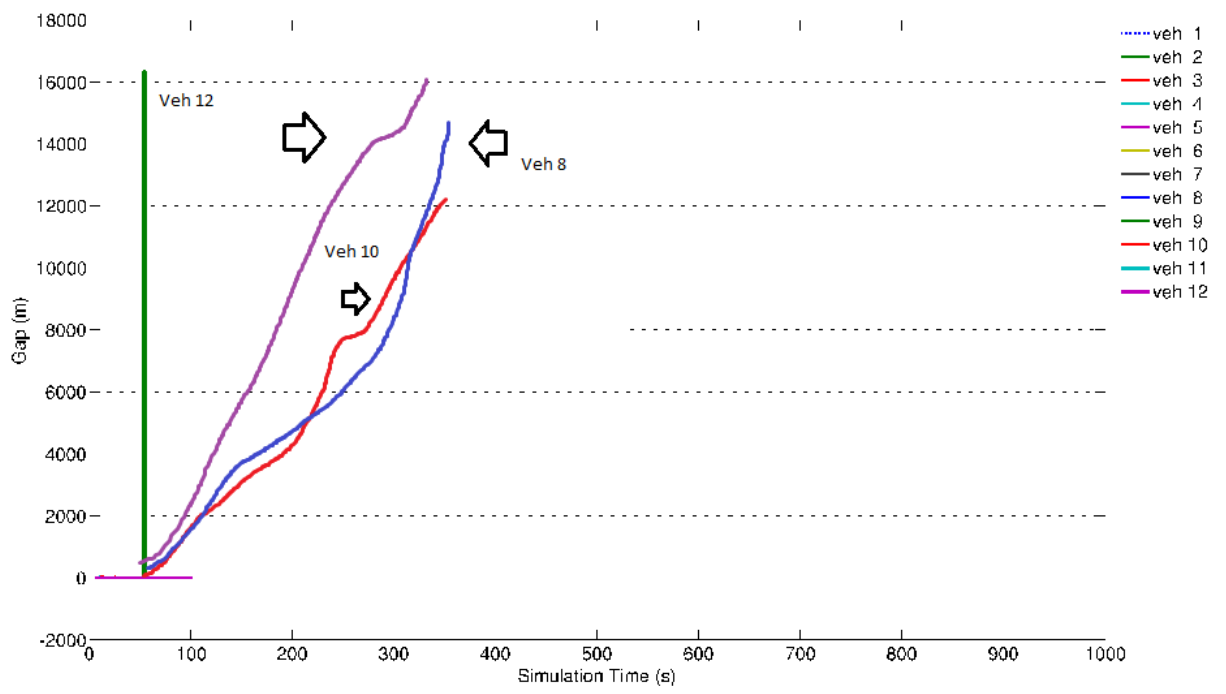


In the figure 4, a plot is shown for 12 vehicles of different types, with an intermediate gap and distance between them. The plot has been restricted with iterations of simulation and it is found that, if the intermediate gap of all 12 types of vehicles are considered, then with available training scheme only vehicle number 12, 8 and 10 can be referred for effective prediction. The other vehicles cannot be considered with this training function, as the intermediate gaps are uncertain and random. The curve shows in the plot are also not smooth and it becomes more stiffer for the best convergence of vehicle 12 .

We demonstrate final results as statistical comparisons. It is the impact of vehicle density and average connectivity distance. We assume statistical Rayleigh fading with superimposed log normal scale. The results show that both vehicle density and average connectivity increases as the average vehicle density increases. Further, as shadow fading occurs, therefore, standard deviation value increases for both these parameters. This means that the average vehicle density required to satisfy a given value of average connectivity distance decreases, whenever the value of standard deviation increases. Three vehicles 12, 8 and 10 are considered to test the convergence of decision in uncertainty, and the lower red curve demonstrates minimum deviation, but with minimum fuzzy training value.

For immediate reference, we present a snapshot of the results as in Table 3: all pairs of iterations with acknowledgment and delivery of movement prediction are shown and minimum error should correspond to greater precision. It should be mentioned that, we performed a single partial derivative to obtain the final training function $T(w)$ with the auxiliary function shown in section 3.1. A higher order and more iterations of partial derivative will lead to better precision and could reduce the error value in prediction.

Table 3: Results for Vehicle Movement

\begin{tabular}{|c|c|c|c|c|c|}
\hline Iteration & Min & Max & Val. passed in function & RMS & Err. \\
\hline \hline Ackn. & 2.1 & 2.6 & 05 & 32.7 & 4.7 \\
\hline Delv. & 1.0 & 7.9 & 15 & 33.6 & 8.7 \\
\hline Ackn. & 5.3 & 8.0 & 20 & 41.2 & 3.92 \\
\hline Delv. & 2.4 & 2.6 & 25 & 34.3 & 2.1 \\
\hline
\end{tabular}


Figure 5: Optimal Vehicle Density \& Connectivity

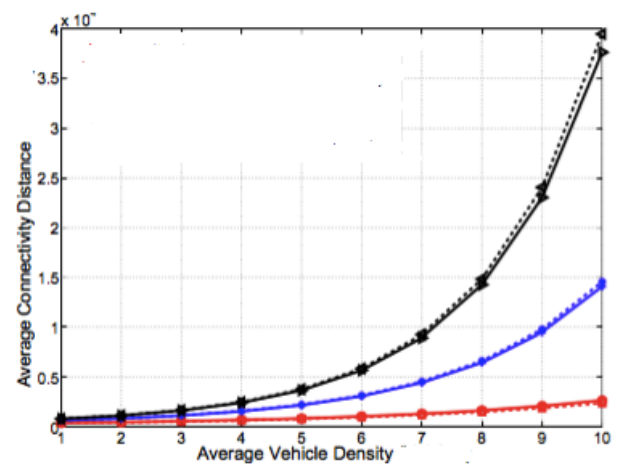

\section{Conclusion}

The paper demonstrated a novel model to predict the movement of vehicles under uncertainty conditions. The approach is implemented through a conventional Boltzmann Machine and trained with fuzzy logic and encompassing the features of a vehicular network. The hidden features and their combinations are expressed as a fuzzy triangular function and thus a computationally lightweight application could be developed. However, while deploying the simulation, it was observed that as conventionally a Boltzmann machine is used for deep learning applications (principally pattern recognition), existing Python libraries are inadequate to support the simulation. The application can be well extended with more real life data instances and if the order of partial derivation could be higher when choosing final training function, better throughput and accuracy could be obtained. A greater numbers of intelligent optimization algorithms like different variants of swarms can be chosen to select the precise combinations of 
parameters. In addition, the complexity of the program may lead to a trade-off between accuracy of prediction and execution time.

\section{References}

[1] H. Omar, W. Zhuang, A. Abdrabou, and L. Li, Performance evaluation of vemac supporting safety applications in vehicular networks, IEEE Trans. Emerg. Topics Comput., vol. 1, no. 1, pp. 69-83, Jun. 2013.

[2] S.Pack and Y.Choi, Fast Handoff Scheme Based on Mobility Prediction in Public Wireless LAN Systems, in IEEE Proceedings- Communications, ,Vol. 151, Issue 5, pp. 489-495, 2004.

[3] G.Yavas, D.Katsaros, O.Ulusoy, and Y. Manolopoulos, A Data Mining Approach for Location Prediction in Mobile Environments, in Data \& Knowledge Engineering, August 2005, Vol.54, Issue 2, pp. 121-146.

[4] X. Wang, C. Wang, G. Cui, and Q. Yang, Practical link duration prediction model in vehicular ad hoc networks, Int. J. of Dist. Sensor Networks, vol. 2015.

[5] Weigang Wu, Jiebin Zhang, Aoxue Luo, Jiannong Cao, Distributed Mutual Exclusion Algorithms for Intersection Traffic Control, IEEE Transactions on Parallel and Distributed Systems, Vol: 26, Issue: 1, Jan. 2015, pp.65 74, 2015.

[6] Jialang Cheng, Weigang Wu, Jiannong Cao, Keqin Li, Fuzzy Group-Based Intersection Control via Vehicular Networks for Smart Transportations, IEEE Transactions on Industrial Informatics,Vol: 13, Issue: 2, April 2017, pp.751-758, 2017.

[7] Nizar Alsharif, Khalid Aldubaikhy and Xuemin Shen, Link Duration Estimation using Neural Networks based Mobility Prediction in Vehicular Networks, IEEE Canadian Conference on Electrical and Computer Engineering (CCECE), IEEE procedings, 2016.

[8] Bingnan Jiang, Yunsi Fei, Traffic and vehicle speed prediction with neural network and Hidden Markov model in vehicular networks, iEEE Intelligent Vehicles Symposium (IV), 2015 .

[9] Thomas Streubel, Karl Heinz Hoffman, Prediction of driver intended path at intersections, IEEE Intelligent Vehicles Symposium Proceedings, 2014.

[10] Hinton, G. E.; Sejnowski, T. J. D. E. Rumelhart, J. L. McClelland, and the PDP Research Group, eds. Learning and Relearning in Boltzmann Machines, 1986.

[11] Hinton, G. E.; Osindero, S.; Teh, Y. A fast learning algorithm for deep belief nets Neural Computation. 18 (7): pp.1527-1554, 2006. 
[12] Ratul Mahajan, CRAWDAD dataset microsoft/vanlan (v. 2007-09-14), downloaded from http://crawdad.org/microsoft/vanlan/20070914, https://doi.org/10.15783/C7FG6S, Sep 2007.

[13] I. Tal, G. M. Muntean, User oriented fuzzy logic based clustering scheme for vehicular Ad hoc networks, IEEE Vehicular Technology Conf. (VTC Spring), pp. 1-5, 2013.

[14] Bevish Jinila, Komathy Rough Set Based Fuzzy Scheme for Clustering and Cluster Head Selection in VANET ELEKTRONIKA IR ELEKTROTECHNIKA, ISSN 1392-1215, Vol. 21, No. 1, 2015.

[15] Miki Aoyagi Learning Coefficient in Bayesian Estimation of Restricted Boltzmann Machine, Journal of Algebraic Statistics Vol. 4, No. 1, pp. 31-58, 2013. 


\section{APPENDIX}

Code Segment:

Communication Prototype Functions */

\#include $<$ mspnvehicle.h $>$

\#include $<$ killApp.h $>$

\#include < boost/thread/thread.hpp>

void openNewTerminal ();

bool notificationKilledProcess

(atv_acrosser : : killApp : : Request \&req,

void receiveKillCommunication(int argc, char $* * \operatorname{argv})$;

boost:: mutex mtxTerminal;

boost : : mutex:: scoped_lock lock (mtxTerminal);

boost: : condition_variable condTerminal;

* It proves the presence of

terminal window that execute the process

communication */

bool existTerminal $=$ false;

/ $* * * * * * * * * * * * * * * * * * * * * * * * * * * * * * * * * * * * * * * * * * * * * * * * * * * * * * * * * * * * * * * * * *$

*@function: openNewTerminal

* Thread opens a new terminal and executes the communication

* program. It also remains waiting status on the condition variable

* to launch again the process communication.

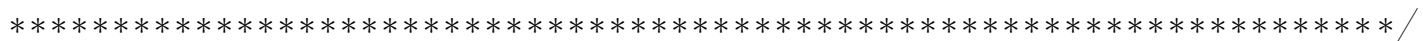

void openNewTerminal ()

\{

int statusSystem $=0$;

*Open the first terminal with communication program*/

existTerminal $=$ true;

statusSystem $=$ system ( gnome- terminal $_{\llcorner}-x_{\downarrow} . /$ communication" $)$;

printf ( $\backslash$ nTERMINAL_OPENED_STATUS: „\%d" , stat us System );

* Infinite while, there will be always a condition variable

which wait a signal from a killed process.

When the the condition variable will be awake from a killed process, it will open a new terminal and execute the communication

program and wait again another signal from a killed process */

while (1)

$\{/$

* Condition variable, wait to be awake after the killed process */

while (existTerminal = true) condTerminal.wait (lock);

/ Open a new terminal and execute the communication process */

statusSystem $=$ system ( gnome-terminal $-x_{\llcorner} . /$communication" $)$;

printf ( $\backslash$ nTERMINAL_OPENED_STATUS: „\%d" , stat us System );

if ( statusSystem < 0 )

p r in t $\mathrm{f}$ ( $" \backslash \mathrm{n} \_P R O B L E M \_T O \_O P E N \_T H E \_N E W \_W I N D O W \_D U R I N G \_T H E$

RESTARTING_OF_THE_SOFTWARE_communication " ) ; 


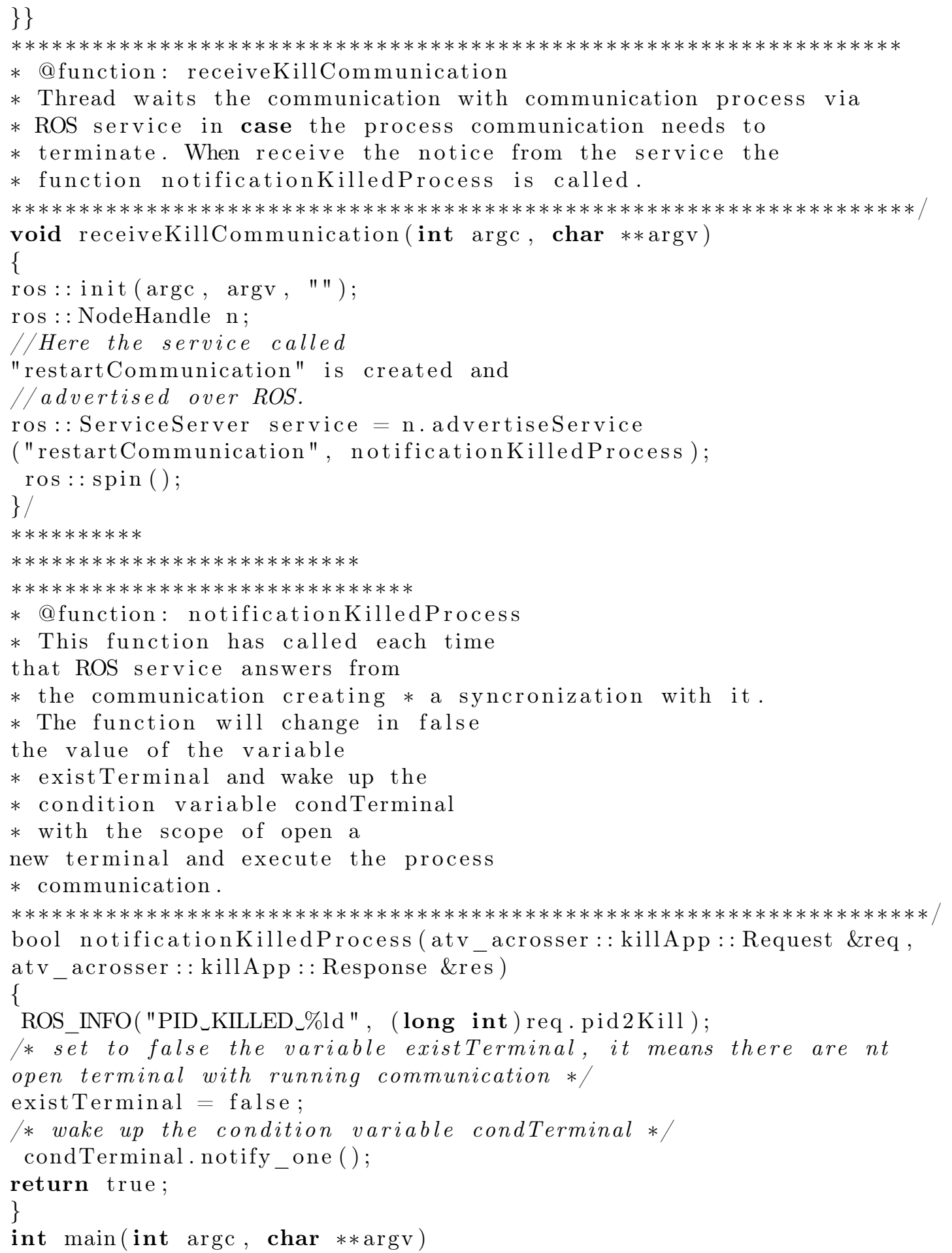




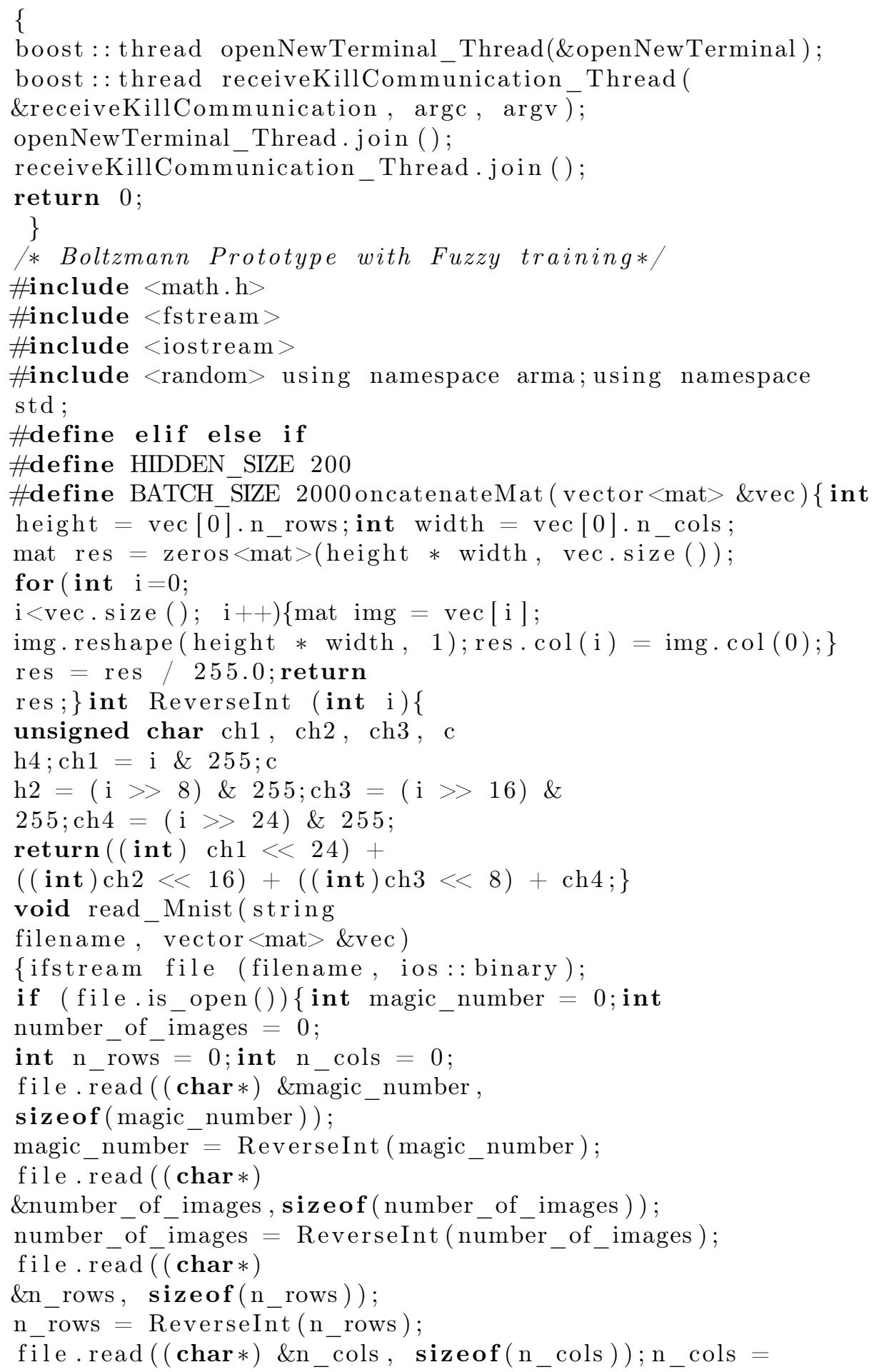




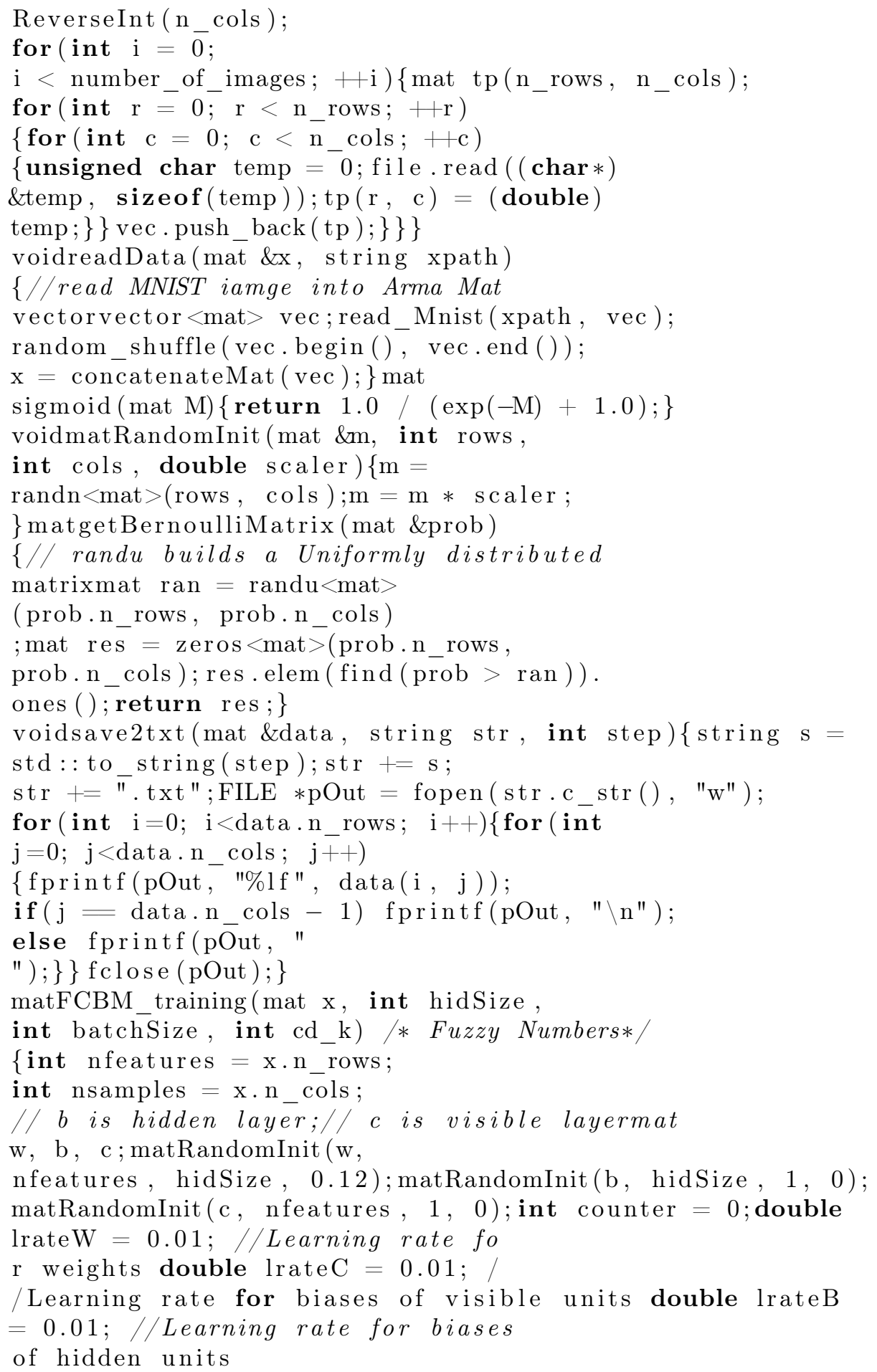




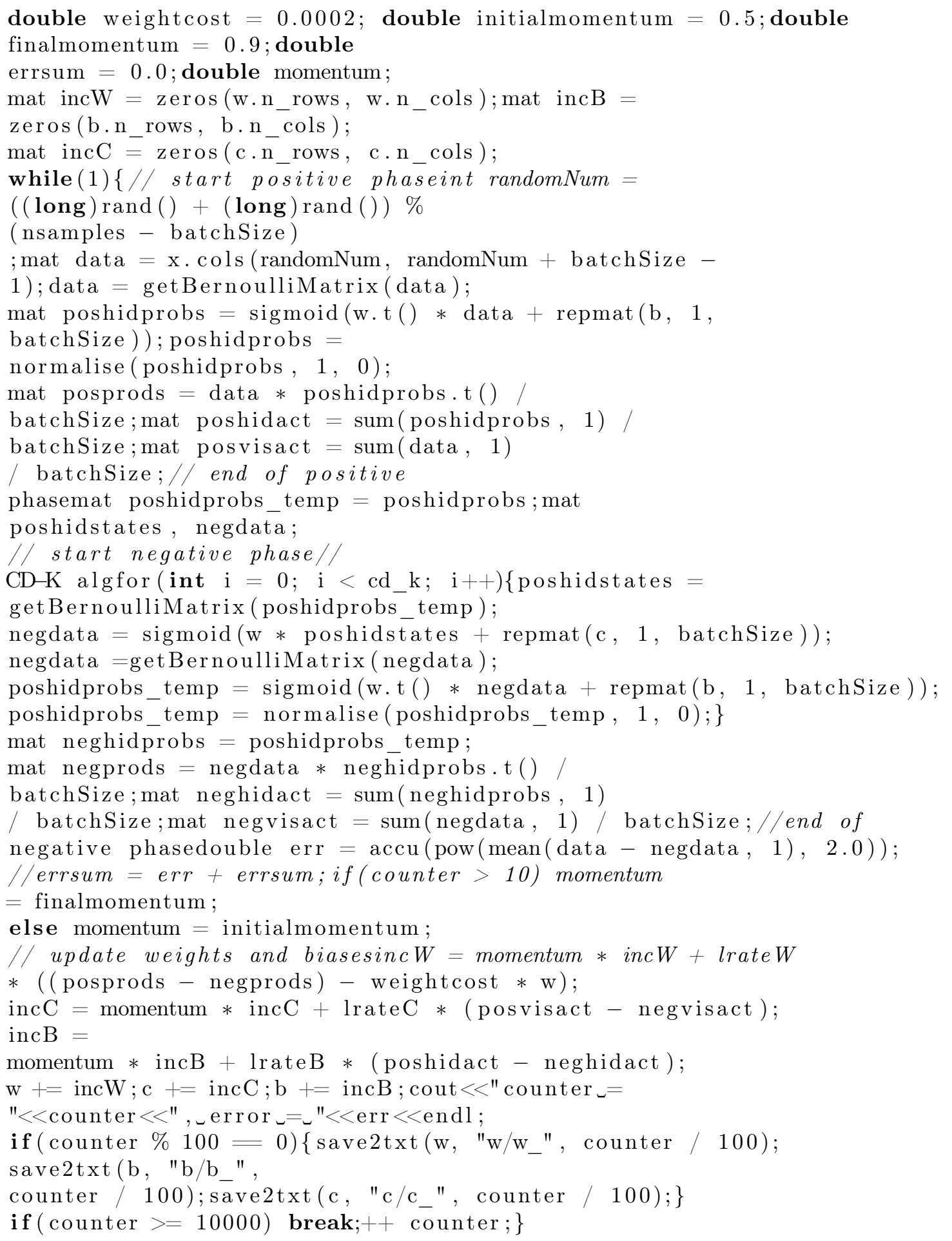




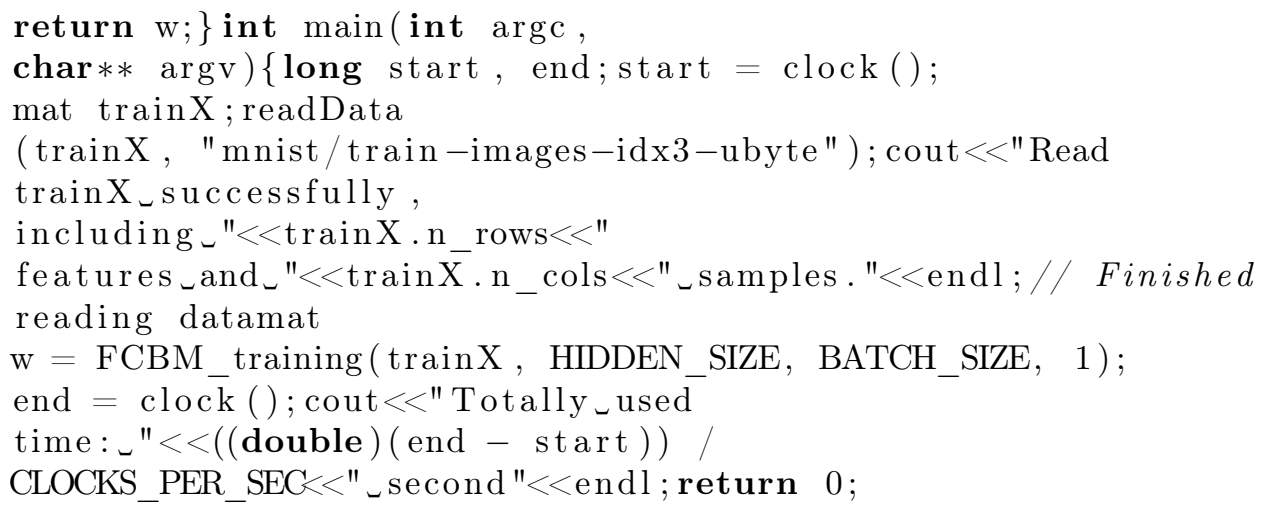

